\title{
ORIENTACIÓN PREFERENCIAL DE FRUTOS Y SU REMOCIÓN EN Myrtillocactus schenckii (CACTACEAE) EN UNA ZONA SEMIÁRIDA INTERTROPICAL
}

\author{
TESIS \\ PARA OBTENER EL GRADO ACADÉMICO DE \\ MAESTRO EN BIOLOGÍA \\ PRESENTA
}

Biól. Leonardo Paz Herrera

Codirector: Dr. Pedro Luis Valverde Padilla

Codirector: $\mathrm{M}$ en C. Gerardo López Ortega

Asesor: Dr. Pablo Corcuera Martínez del Río

MÉXICO D.F.

DICIEMBRE 2016 
“La Maestría en Biología de la Universidad Autónoma Metropolitana pertenece al Programa Nacional de Postgrados de Calidad del CONACyT, con la categoría de Consolidado" 
El jurado designado por la

\section{División de Ciencias Biológicas y de la Salud}

de la Unidad Iztapalapa aprobó la tesis que presentó

\section{BIÓL. LEONARDO PAZ HERRERA}

\section{El día 2 de diciembre del año 2016}

\section{Comité Tutoral y Jurado}

Codirector: Dr. Pedro Luis Valverde Padilla

Codirector: M. en C. Gerardo López Ortega

Asesor: Dr. Pablo Corcuera Martínez del Río

Sinodal: M. en C. Fernando Vite González

Sinodal: Dr. Miguel Ángel Armella Villalpando

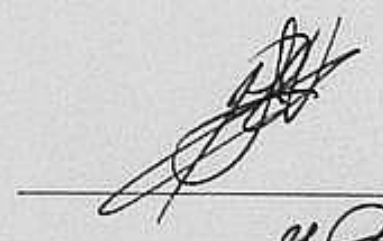

Sinodal: Dr. Juan Héctor García Chávez

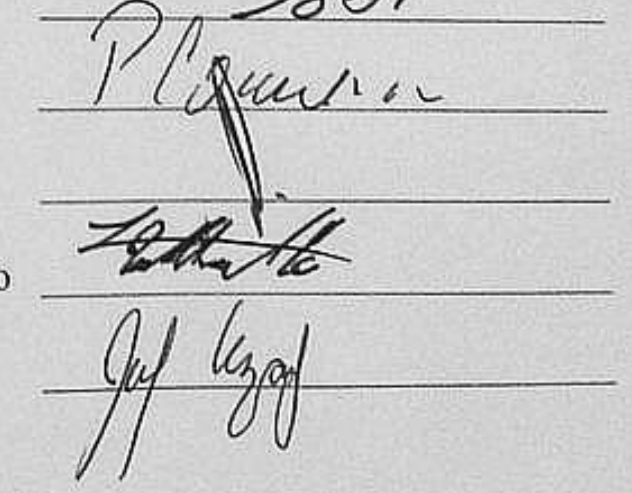


La ciencia es una empresa cooperativa, que se extiende de generación en generación. Es el paso de la antorcha del maestro, al estudiante, al maestro... Una comunidad de mentes que se remonta a la antigüedad y hacia adelante, a las estrellas.

Neil deGrasse Tyson, astrofísico y divulgador de la ciencia. 


\section{AGRADECIMIENTOS}

A la Maestría en Biología de la Universidad Autónoma Metropolitana, por abrirme las puertas y brindarme la oportunidad de seguir con mi formación científica.

Al Consejo Nacional de Ciencia y Tecnología (CONACyT) por las facilidades otorgadas. Es muy difícil dedicarse a la ciencia en una cultura que aún no aprende a valorar el saber.

A mi Comité Tutoral: Dr. Pedro Luis Valverde Padilla, M. en C. Gerardo López Ortega, y Dr. Pablo Corcuera Martínez del Río por aceptarme cuando otros investigadores decidieron que no era idóneo para un postgrado.

A los profesores M en C. Fernando Vite González, Biol. Marco Aurelio Pérez Hernández, a las M en C. y futuras científicas Arizbe Ponce y Sandra Aguilar, así como al guía Pedro Guadalupe Miranda Pacheco por su apoyo y esfuerzo en el trabajo de campo.

A la familia Vera Medina por permitirme realizar el trabajo de campo y hacerle un espacio en su hogar a un desconocido. Muchas gracias Silvia, Isaí, Bartola, Dina y Mehida.

A la Dra. Blanca Pérez García, al Dr. Miguel Ángel Armella Villalpando y al Dr. Juan Héctor García Chávez por sus consejos para la elaboración de la tesis y la presentación del examen profesional. 


\section{AGRADECIMIENTOS ESPECIALES}

A mi mamá (Cecilia Herrera) por su cariño y cuidados, a mi papá (Héctor Paz) por ser ejemplo de trabajo y esfuerzo, a mis hermanos Oscar y Héctor por la compañía durante muchos años; aunque me mudé, la familia la llevo conmigo. A mis abuelas Cecilia y Gloria que siempre velan por la familia, a mis tías Hortensia y Araceli por alentar ese espíritu científico que todo niño tiene. A mis sobrinas Cecilia, Ana y Lilith por su alegría, cariño, sinceridad y por darme la oportunidad de ser una mejor persona.

A mis roomies Carlos y Alejandro, en esta nueva experiencia de dejar el nido familiar, así como a los visitantes por compartir sus aventuras de la vida y hacer de Ñoño House un hogar feliz: Allan, Obed, Isaac, Jazmín, Sandra, Vania y Zenia. Viva la libertad.

A Luz y Sandra, biólogas de mente, corazón y alma, mujeres ejemplares que luchan por vivir el sueño; desde la FESI hasta el horizonte, gracias por todos estos años y los demás que faltan. Iztacalos por siempre.

A los Ñoños del Mal: Alejandro, Jessica, Laura y Yazmin, gracias por hacer de mi breve estancia en la UAMI un recuerdo feliz, sin ustedes no sería lo mismo; así como a Ivonne, Karen, Luis, Marlene, Sara y Thalia por toda su buena vibra y sus consejos. Nos vemos en el doctorado. 


\section{ÍNDICE}

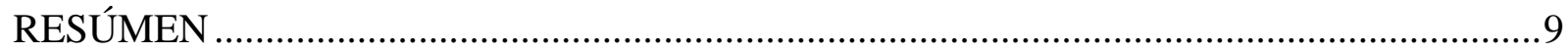

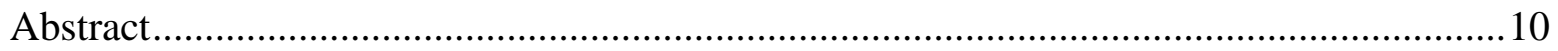

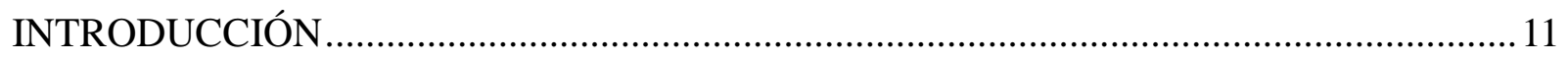

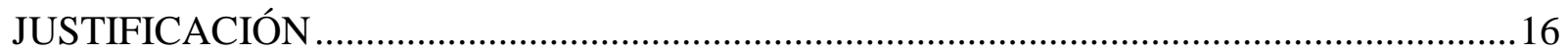

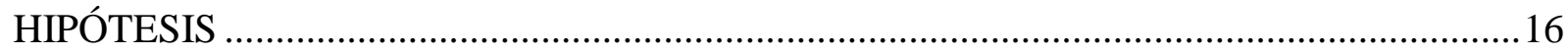

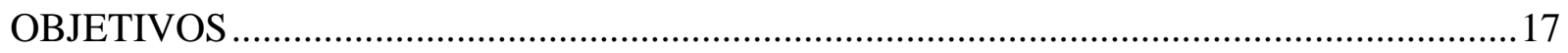

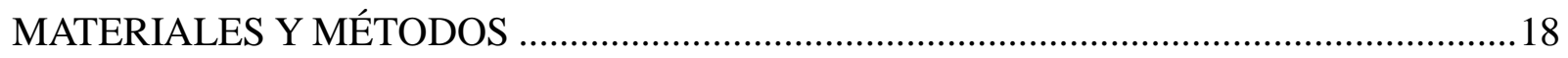

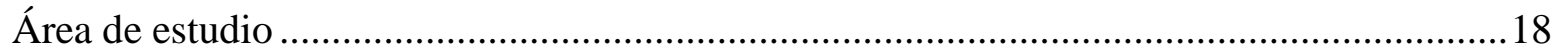

Myrtillocactus schenkii (J. A. Purpus) Britton y Rose, Contr. ................................................19

Determinación de la distribución circular de los frutos..........................................................21

Experimentos de remoción de frutos por consumidores diurnos y nocturnos..........................23

Identificación de las aves que consumen frutos de Myrtillocactus schenckii ........................25

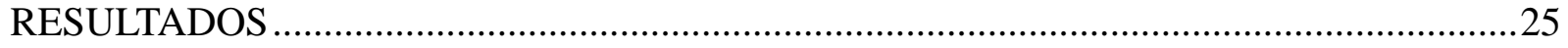

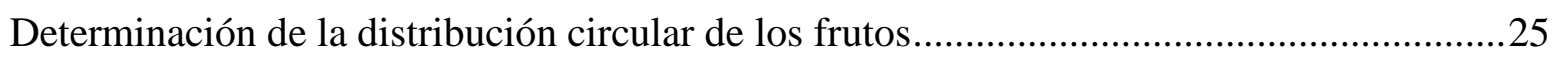

Experimentos de remoción de frutos por consumidores diurnos y nocturnos.........................30

Identificación de las aves que consumen frutos de Myrtillocactus schenckii ........................32

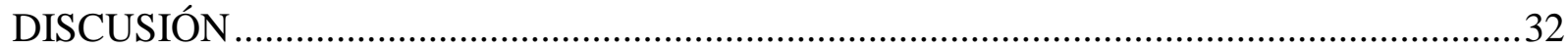

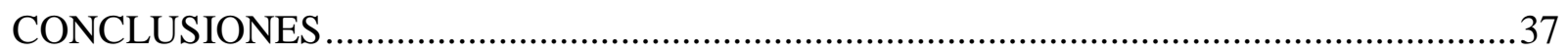

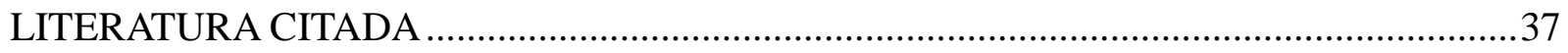




\section{ÍNDICE DE FIGURAS}

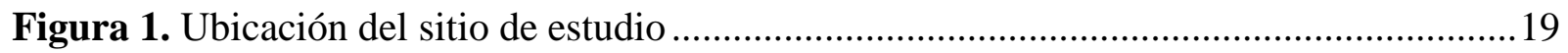

Figura 2. Myrtillocactus schenckii y sus estructuras reproductivas ..........................................21

Figura 3. Conteo de estructuras de Myrtillocactus schenckii ....................................................22

Figura 4. Rama de un individuo de Myrtillocactus schenckii cubierta durante el día, acompañada por una trampa para frutos que cayeran................................................................2.

Figura 5. Distribución de frecuencias circulares de yemas florales y flores de Myrtillocactus

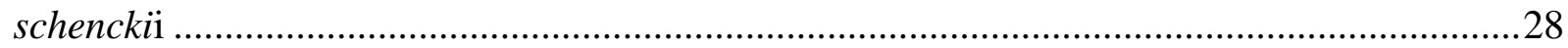

Figura 6. Distribución de frecuencias circulares de frutos de Myrtillocactus schenckii ..........29

Figura 7. Proporción de frutos removidos por consumidores diurnos y nocturnos de Myrtillocactus schenckii

\section{ÍNDICE DE TABLAS}

Tabla 1. Estadísticos circulares básicos y prueba de uniformidad de las orientaciones de las yemas florales y flores de Myrtillocactus schenckii .26

Tabla 2. Estadísticos circulares básicos y prueba de uniformidad de las orientaciones de los frutos de Myrtillocactus schenckii.

Tabla 3. Número total de frutos registrados por sectores en 40 individuos de Myrtillocactus schenckii.

Tabla 4. Resultados de los experimentos de remoción de frutos por consumidores diurnos y nocturnos de Myrtillocactus schenckii .31

Tabla 5. Especies de aves que consumen los frutos de Myrtillocactus schenckii. .32 


\section{RESÚMEN}

La orientación ecuatorial de frutos se presenta en cactáceas diversas. En algunas especies del hemisferio norte, se ha reportado que los frutos producidos en las ramas y costillas sureñas son más grandes y contienen más semillas. Sí estos son más atractivos para los frugívoros, esperamos que muestren una preferencia por ellos en comparación con los producidos en las ramas y/o costillas norteñas. Para probar esta hipótesis, se estudió el caso de Myrtillocactus schenckii (observaciones preliminares, sugirieron una distribución preferencial). En el presente estudio se investigó 1) si la cactácea muestra orientación preferencial en la producción de frutos, 2) si los consumidores muestran una preferencia por los frutos sureños independientemente de su disponibilidad, y 3) se evaluó sí existen diferencias en la remoción entre consumidores diurnos contra nocturnos. Para describir la distribución circular, se eligieron 40 individuos, en los cuales la copa se dividió en dos sectores (norte y sur), se eligieron 2 ramas por cada sector (las que se encuentren más orientadas a su respectivo sector), en donde se registró la orientación de cada costilla y se contaron las diferentes estructuras reproductivas (yema, flor y fruto). Para los experimentos de remoción, se tomaron 20 individuos; para cada uno se seleccionó una rama donde se marcó un número determinado de frutos maduros. Durante tres días en el 2014, los frutos de 10 individuos fueron expuestos a los frugívoros diurnos (333 y 251 frutos en abril y mayo, respectivamente) y 10 a los nocturnos (490 y 287, respectivamente). En abril del 2015 se expusieron los frutos de 5 individuos durante el día y otros 5 en la noche (175 y 156, respectivamente). Para las exclusiones, se utilizaron bolsas de paño de cielo (tul) y se colocaron trampas para frutos. Los frutos presentaron una marcada orientación hacia el sur $\left(\mu=185.5^{\circ}\right)$, con 2.2 frutos sureños por cada norteño. La notable carencia de frutos norteños no permitió evaluar las preferencias de los frugívoros. Sólo se removieron el $14 \%$ de los frutos y no se detectaron diferencias significativas entre diurnas y nocturnas para el 2014 ( $p$ < 0.05), pero en el 2015 ocurrió una mayor remoción durante la noche. Por las características de sus frutos (forma, color, posición, carbohidratos, y otros), M. schenckii es consumida preferentemente por aves (6 especies, destacando una granívora y dos frugívoras), no obstante, los consumidores nocturnos fueron los removedores más importantes. 


\section{ABSTRACT}

The equatorial orientation of fruits is present in diverse cacti. In some species from the north hemisphere, have reported they produce bigger fruits and more seeds in southern branches and/or ribs. If these are more attractive for frugivores, we expect them show a preference for them in comparison with those produced in northern branches and ribs. To prove this hypothesis, the case of Myrtillocactus schenckii was studied (preliminary observations suggest a preferential distribution). This study investigated 1) if the cacti shows preferential orientation in fruit production, 2) if consumers shows a preference for southern fruits independently of their availability, and 3) if differences existed in removal between diurnal and nocturnal consumers. To describe the circular distribution, 40 individuals was selected, the cups was divided in two sectors (north and south) with two branches per each sector (those with a clear orientation to its respective sector), where the orientation of each branch was registered, and the different reproductive structures (bud, flower, fruit) were counted. For removal experiments, 20 individual was selected; one branch of each individual was chosen and a determined number of its mature fruits were marked. For three days on 2014, the fruits of 10 individuals were exposed to diurnal frugivores (333 and 251 fruits in April and May, respectively) and 10 individuals for nocturnal (490 and 287, respectively). On April of 2015 the fruits of 5 branches has exposed in the day and another 5 for the night (175 and 156, respectively). For exclusions, bags of tulle netting were used and fruit traps were placed. The fruits present a marked orientation towards the south $\left(\mu=185.5^{\circ}\right)$, with 2.2 southern fruits per each northern fruit. The notable lacks of northern fruits didn't allow evaluation preferences of frugivores. Only 14\% of fruits were removed and significant differences between diurnal and nocturnal in 2014 was not detected ( $<$ 0.05), but on 2015, a major removal during the night was occurred. Because the features of their fruits (form, color, position, carbohydrates, and others), M. schenckii is preferably consumed for birds (6 species, highlighting one granivore and two frugivores), however, the nocturnal consumers were the most important removers. The differences between diurnal and nocturnal removal in both years can be caused for differences in the precipitation during the rainy season. 


\section{INTRODUCCIÓN}

Las cactáceas (Familia Cactaceae) son plantas autóctonas de América, distribuidas desde Canadá hasta la Patagonia, en Argentina, así como en altitudes desde el nivel del mar en las dunas costeras, hasta los 5100 m s.n.m. (Bravo-Hollis, 1978; Bravo-Hollis y Scheinvar, 1995; Bustamante y Búrquez, 2005). Aunque esta familia muestran una amplia distribución, están mejor representadas en las regiones áridas y semiáridas (Arakaki, et al., 2011). La familia Cactaceae está compuesta por alrededor de 125 géneros (Anderson, 2001, Arias-Montes et al., 2012) y 1850 especies (Nyffeler y Eggly, 2010). Esta gran diversidad es resultado de uno de los sucesos de radiación más notables registrados en las zonas áridas del mundo (Edwards et al., 2005, Arakaki et al., 2011). La familia Cactaceae posee como centros de alta diversidad a México, el suroeste de los Estados Unidos, la región de los Andes centrales, en Perú y Bolivia, y el este de Brasil (Arakaki et al., 2011). Muestra una gran diversidad de formas, tamaños y hábitos de crecimiento (Gibson y Nobel, 1986). México, por sus peculiares condiciones de latitud, topografía y climas, es el país que alberga la mayor cantidad de especies, contando con 60 géneros y 670 especies, de las cuales 77\% son endémicas (Bravo-Hollis, 1978; AriasMontes et al., 2012); y en el caso del Valle de Tehuacán-Cuicatlán, región donde se realizó el presente estudio, habitan 28 géneros con 86 especies (Arias-Montes et al., 2012).

Las zonas áridas y semiáridas son las regiones del planeta que se caracterizan por la escasa disponibilidad de agua, así como por los altos niveles de insolación durante la mayor parte del año (Noy-Meir, 1973). En México están definidas como zonas donde la precipitación es del orden de $250 \mathrm{~mm}$ anuales o menos, y como semiáridas a aquellas en donde la precipitación oscila entre más de 250 y menos de 500 mm anuales, ambas influenciadas por la 
temperatura y la altitud (González-Medrano, 2012). En México, estas regiones representan cerca del 60\% del territorio (Rzedowski, 1993), siendo el matorral xerófilo el tipo de vegetación dominante en esas zonas (Rzedowski, 1978). Las Cactáceas son una parte importante y característica del paisaje de estas zonas y en algunos casos son el componente dominante de la vegetación (Bravo-Hollis, 1978; González-Medrano, 2012).

Las Cactáceas son reconocidas por desplegar una gran diversidad de estructuras y mecanismos muy especializados (Gibson y Nobel, 1986; Nobel, 1998). Este conjunto de estructuras y mecanismos son considerados adaptaciones (Edwards y Donoghue, 2006) que han evolucionado en respuesta a las condiciones de déficit de agua y altas temperaturas existentes en los hábitats áridos y semiáridos (Gibson y Nobel, 1986; Nobel y Loik, 1999; Edwards et al., 2005 Edwards y Donoghue, 2006; Valverde et al., 2007). Además de la suculencia, quizá la característica más evidente en las cactáceas (Gibson y Nobel, 1986), poseen otras adaptaciones sobresalientes como la cubierta de espinas, la pubescencia apical o lateral (Gibson y Nobel, 1986; Nobel y Loik, 1999), raíces someras, cutícula cerosa (Edwards y Donoghue, 2006; Arakaki et al., 2011) y la vía de fotosíntesis tipo CAM (Metabolismo ácido de las Crassuláceas, por sus siglas en inglés) (Gibson y Nobel, 1986; Nobel y Loik, 1999; Edwards y Donoghue, 2006; Arakaki et al., 2011).

La orientación no-aleatoria o preferencial, con respecto al ecuador, en la que se encuentran distribuidas las estructuras reproductivas tales como yemas, flores y frutos, es otra característica especializada presente en algunas cactáceas columnares y arborescentes con floración subapical y lateral (Johnson, 1924; Zavala-Hurtado et al., 1998; Tinoco-Ojanguren y Molina-Freaner, 2000; Figueroa-Castro y Valverde, 2011; Aguilar-Gastelum y Molina-Freaner, 2015). Al respecto, se ha reportado un acimut preferencial de las estructuras reproductivas 
hacia el ecuador (orientación ecuatorial), distribuyéndose con mayor frecuencia en las caras del tallo o ramas que experimentan mayor exposición a la radiación solar, así como, temperaturas más elevadas (Nobel, 1981; Gibson y Nobel, 1986; Zavala-Hurtado et al., 1998; Nobel y Loik, 1999; Tinoco-Ojanguren y Molina-Freaner, 2000). Lo anterior se ha planteado como una estrategia que permite optimizar la intercepción de radiación fotosintéticamente activa (RFA), favoreciendo la captura de $\mathrm{CO}_{2}$ (Geller et al., 1986; Tinoco-Ojanguren y Molina-Freaner, 2000) y exponer a las yemas florales a una temperatura propicia para el desarrollo de las flores (Johnson, 1924; Zavala-Hurtado et al., 1998; Tinoco-Ojanguren y Molina-Freaner, 2000; Herce et al., 2014; Aguilar-Gastelum y Molina-Freaner, 2015). En México, este patrón de orientación ecuatorial se ha reportado en Myrtillocactus geometrizans (Rosas-García, 2010; Valencia-Mendoza, 2014), Pachycereus weberi (Córdova-Acosta, 2011), P. pringlei (Tinoco-Ojanguren y Molina-Freaner, 2000) y P. pecten-arboriginum (AguilarGastelum y Molina-Freaner, 2015).

En su estudio con P. pringlei, Tinoco-Ojanguren y Molina-Freaner (2000) proponen una hipótesis como explicación para este patrón de orientación ecuatorial de estructuras reproductivas: la intercepción de radiación fotosintéticamente activa (RFA) es el factor responsable por sus efectos sobre la captura de dióxido de carbono $\left(\mathrm{CO}_{2}\right)$ y la temperatura del tallo. Los autores plantean una ganancia de carbono diferencial entre costillas con distinta orientación y que no existe translocación de recursos entre ellas. Si la inducción de las areolas para la producción de yemas florales depende de la acumulación de carbohidratos, únicamente aquellas costillas con orientación ecuatorial acumularán los suficientes carbohidratos necesarios para la producción de flores y frutos.

En muchas regiones áridas y semiáridas, en donde las cactáceas columnares poseen 
una amplia distribución, como es el caso de la región de Tehuacán-Cuicatlán existe una estrecha relación mutualista entre estos y varias especies de aves y murciélagos (ValienteBanuet et al., 1996). Particularmente para las aves frugívoras y granívoras, los frutos de las cactáceas columnares pudieran representar recursos alimenticios importantes. De hecho, muchos frutos de cactáceas columnares presentan el síndrome de ornitocoria, que implica características para ser removidos por aves (Pérez-Villafaña y Valiente-Banuet, 2009). En este sentido, las aves frugívoras eligen frutos dulces y la variación del azúcar ocurre naturalmente entre especies frutales (Levey, 1987). Además del contenido calórico, los frutos rojos son llamativos para las aves (Welty, 1982), color que, en las cactáceas, es resultado de pigmentos llamados betalainas (Robinson, 1963; Hernández-López et al., 2008), además de las aves, se ha reportado que otros vertebrados son atraídos por frutos carnosos ricos en agua y azúcares (Montiel y Montaña, 2000).

Estudios recientes han aportado evidencia que sugiere que el patrón de orientación ecuatorial tiene efecto sobre algunas de las características de flores y frutos. Por ejemplo, en $P$. weberi, Córdova-Acosta (2011) reportó que las flores con orientación sureña presentan mayor diámetro medio (una medida de su tamaño) y son más exitosas en producir frutos y semillas. En la misma especie, Figueroa-Castro y Valverde (2011) encontraron que las flores con orientación hacia el sur contenían mayor cantidad de óvulos, y los frutos sureños contenían más semillas y de mayor peso. Valencia-Mendoza (2014) reportó que el tamaño de las flores, el número de óvulos por flor y el tamaño del ovario fueron mayores en aquellas flores orientadas hacia el sureste en Myrtillocactus geometrizans. Con base en estas evidencias en cactáceas columnares, es posible que, como consecuencia de la acumulación diferencial de fotosintetatos producto de la variación en la intercepción de RFA experimentado por la planta, 
los frutos producidos en las ramas o costillas con orientación hacia el sur serán más abundantes, más grandes y con mayor contenido de semillas (Figueroa-Castro y Valverde, 2011). Si la abundancia y el tamaño de los frutos, entre otras características, juegan un rol central en la dispersión de las semillas por parte de los frugívoros (Howe y Miriti, 2004), es probable que los frutos producidos en las ramas o costillas con orientación hacia el sur sean más atractivos para las aves granívoras y frugívoras, así como para otros vertebrados.

En el presente estudio se aborda el caso de Myrtillocatus schenckii (J. A. Purpus) Britton \& Rose, Contr., el cual es un cacto arborescente endémico de México (Bravo-Hollis, 1978; Guzmán et al., 2003) cuya distribución se restringe a las regiones semiáridas de Puebla y Oaxaca (Ortiz et al., 2010). Observaciones preliminares indicaron que las estructuras reproductivas (i. e., yemas, flores y frutos) se producían preferentemente en las ramas, y en los lados de las mismas orientados hacia el sur. Esta evidencia sugeriría que era posible que $M$. schenckii también presentara el fenómeno de orientación ecuatorial.

$\mathrm{Al}$ igual que el resto de las especies del género, $M$. schenckii produce frutos pequeños, globosos, de color rojo carmín, carnosos y con pulpa morena-rojiza (Bravo-Hollis, 1978), los cuales son consumidos por aves granívoras y frugívoras, al igual que por hormigas (Ortiz et al., 2010). De presentar M. schenckii el patrón de orientación ecuatorial y de tener este efectos en la abundancia, tamaño y calidad (número de semillas) de frutos, es posible hipotetizar que, tanto las aves como otros vertebrados, mostrarán preferencias por los que se desarrollen en los sectores sur de las plantas, al igual que en las ramas y costillas con orientación también sureña. 


\section{JUSTIFICACIÓN}

En la región de Tehuacán-Cuicatlán se ha investigado el patrón de orientación ecuatorial de estructuras reproductivas de algunas especies de cactáceas columnares (Córdova-Acosta, 2011; Figueroa-Castro y Valverde, 2011, Aguilar-García, 2015), así como sobre la frugivoría y dispersión de frutos por aves en cactáceas (Arizmendi y Espinosa de los Monteros, 1996; Valiente-Banuet et al., 1996; Pérez-Villafaña, 2000; Godínez-Álvarez et al., 2002; PérezVillafaña y Valiente-Banuet, 2009). Sin embargo, no existe evidencia publicada en donde se analice el fenómeno de orientación ecuatorial en M. sckenckii y menos aún, en donde se evalúe si este patrón de orientación no aleatoria de los frutos tiene alguna implicación en el consumo diferencial de frutos por aves y otros vertebrados.

\section{HIPÓTESIS}

Existe evidencia en cactáceas de que estas producen frutos más grandes y con mayor contenido de semillas en las areolas orientadas al sur (Córdova-Acosta, 2011; Figueroa y Valverde, 2011; Valencia-Mendoza, 2014) y, si tales características los hace más llamativos para los frugívoros, se espera que las aves y otros vertebrados muestren una preferencia por los frutos con orientación sur, comparados con los frutos orientados hacia el norte. 


\section{Preguntas de investigación}

1) ¿̇Mrtillocactus schenckii presenta orientación preferencial en la producción de frutos?

2) Si los frutos de $M$. schenckii muestran un patrón de orientación preferencial, ¿las preferencias de los consumidores responden a este patrón (i. e., preferencia por aquellos frutos producidos en las ramas o costillas con orientación hacia el sur)?, y de ser así, ¿¿existen diferencias entre las remociones por consumidores diurnos y nocturnos?

\section{OBJETIVOS}

\section{Objetivo general}

Determinar la distribución circular de los frutos de Myrtillocactus schenckii y las consecuencias en su remoción por aves frugívoras y granívoras, así como por otros vertebrados.

\section{Objetivos particulares}

- Determinar si Myrtillocactus shenckii muestra el fenómeno de orientación preferencial en la producción de frutos.

- Determinar si los consumidores de frutos de Myrtillocactus schenckii tienen preferencia por aquellos producidos en las ramas o costillas con orientación sur.

- Evaluar la importancia de los consumidores diurnos versus los nocturnos en función de su frecuencia de remoción de frutos. 


\section{MATERIALES Y MÉTODOS}

\section{Área de estudio}

El Valle de Zapotitlán, Puebla (18²0' N, 98²8' O), es una cuenca local del Valle de Tehuacán, que forma parte de la Reserva de la Biósfera de Tehuacán-Cuicatlán y de la provincia florística del mismo nombre (Rzedowski, 1978). Se encuentra en la cuenca del río Salado, correspondiente a la parte alta de la cuenca del Papaloapan; situado entre los 1,000 y 1,800 m, con clima semiárido y suelos rocosos y calizos (Bravo-Hollis, 1978). El clima del Valle, basándose en la clasificación de Köppen modificado por García (2006) corresponde a BS 0 wh(e), “seco con régimen de lluvia en verano”, observándose una canícula en la mitad del periodo de lluvias, de mayo a septiembre y mostrando el menor índice de precipitación en julio. La precipitación media anual es de unos $450 \mathrm{~mm}$ y una temperatura anual de $21^{\circ} \mathrm{C}$; la aridez del Valle se debe a la presencia de la Sierra Madre del Sur, que obstruye el paso de los vientos húmedos provenientes del Golfo de México (Valiente-Banuet, 1991).

El tipo de vegetación es de arbustos tropicales áridos, también reportado como selva baja caducifolia espinosa, con matorrales dominantes micrófilos (Bravo-Hollis, 1978, Rzedowski, 1978); esta zona posee una gran riqueza de comunidades, agrupadas en parches que conforman mosaicos de vegetación con un gran número de especies restringidas a cada uno de ellos (Osorio et al., 1996). Los tipos de vegetación principal son caracterizados por la dominancia de cactáceas columnares, con densidades de 1800 individuos/ha (Osorio et al., 1996). El presente estudio se realizó en la cima y ladera del cerro “El Chichipe” (18¹4’06.12” N, 97²9’24.60” O, altitud 1816 m s.n.m.), adyacente a la localidad de Los Reyes Metzontla, del municipio de Zapotitlán, Puebla (Figura 1). 


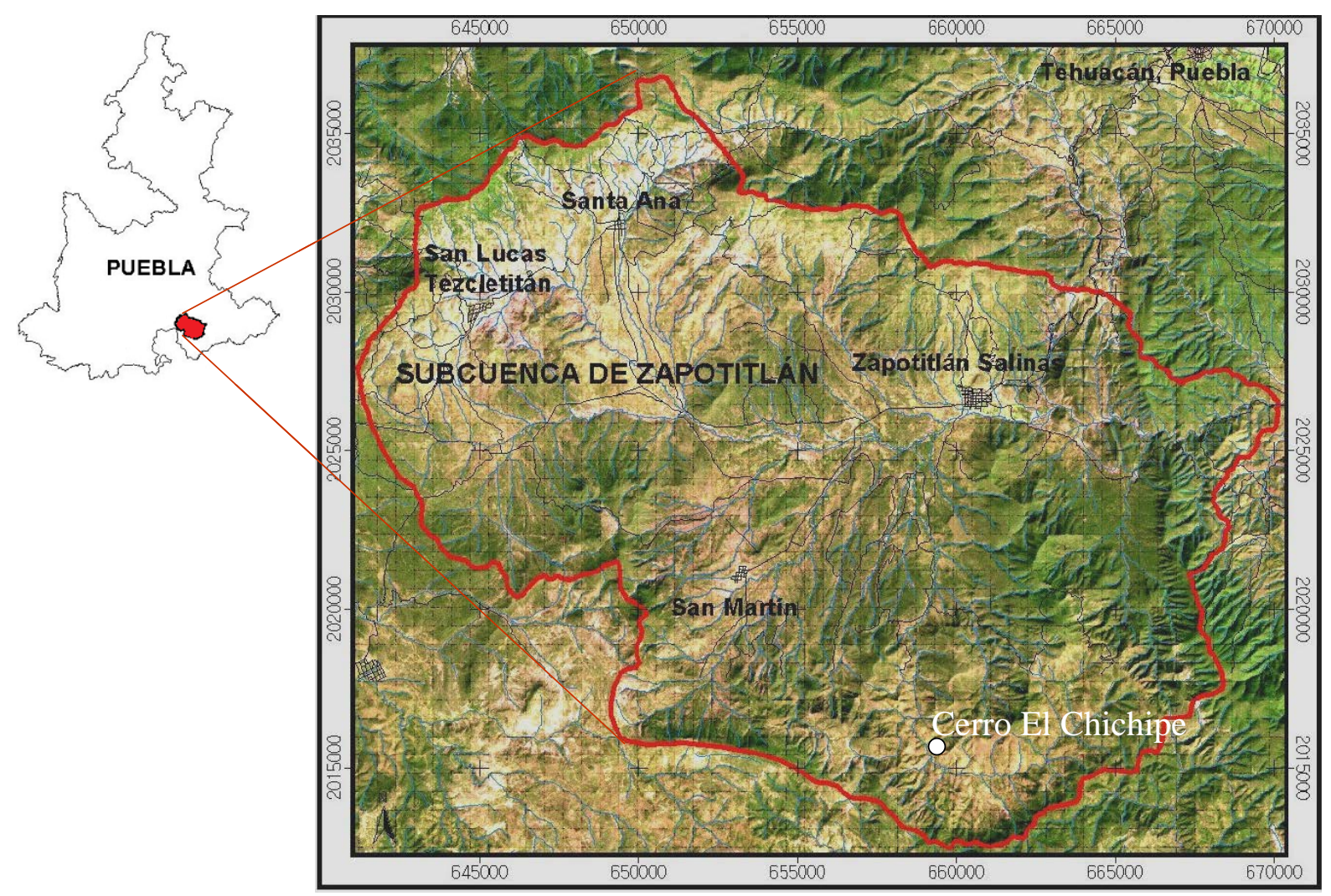

Figura 1. Ubicación del sitio de estudio, cerro "El Chichipe" en los Reyes Metzontla, dentro del Valle de Zapotitlán (sureste del estado de Puebla) en la porción centro-norte de la Reserva

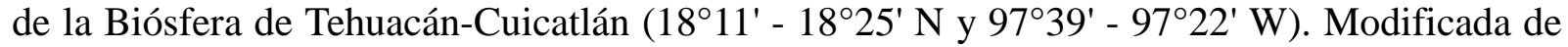
Sandoval-Palacios (2010).

Myrtillocactus schenkii (J. A. Purpus) Britton y Rose, Contr.

Es un cacto arborescente, endémico de las áreas semiáridas de Puebla y Oaxaca, en el centro de México, que crece de forma silvestre en el bosque tropical caducifolio y el matorral xerófilo, en elevaciones de 1200 a 1900 m s.n.m. (Arias-Montes et al., 2012). Es comúnmente llamado "garambullo", alcanza una altura de 3 a 5 m., con ramas de color verde oscuras de aproximadamente $10 \mathrm{~cm}$. de diámetro (Arias-Montes et al., 2012) (Figura 2). La época de floración ha sido reportada de noviembre a enero, en ocasiones hasta abril, mientras que la 
producción de frutos es de mayo a julio (Bravo-Hollis, 1978; Blancas-Vázquez, 2007; AriasMontes et al., 2012). Sus flores son pequeñas (una corola abierta aproximadamente de $2.5 \mathrm{~cm}$. de diámetro), blancas y con antesis diurna, visitadas por especies de insectos como Apis mellifera, Tabanus sp. y Xylocopa mexicanorum (Bravo-Hollis, 1978, Ortiz-Aguilar, 2009). Sus frutos carnosos (bayas) miden de 0.8 a $1.5 \mathrm{~cm}$. de largo y de 0.6 a $1.5 \mathrm{~cm}$. de ancho, globosos o elipsoides, rojos con pulpa de parda a roja, consumidos asiduamente por aves (Bravo-Hollis, 1978; Arias-Montes et al., 2012) (Figura 2), y con aproximadamente 68 semillas por fruto en individuos silvestres (Ortiz-Aguilar, 2009). Los habitantes de la localidad consumen los frutos de esta especie de poblaciones silvestres, silvícolas o cultivadas, ya sea como alimento fresco o procesado en diferentes formas, mientras que sus tallos y ramas son utilizados para el forraje, la leña y como cercas vivas (Blancas-Vázquez, 2007; HernándezLópez et al., 2008; Blancas et al., 2009; Ortiz-Aguilar, 2009). 


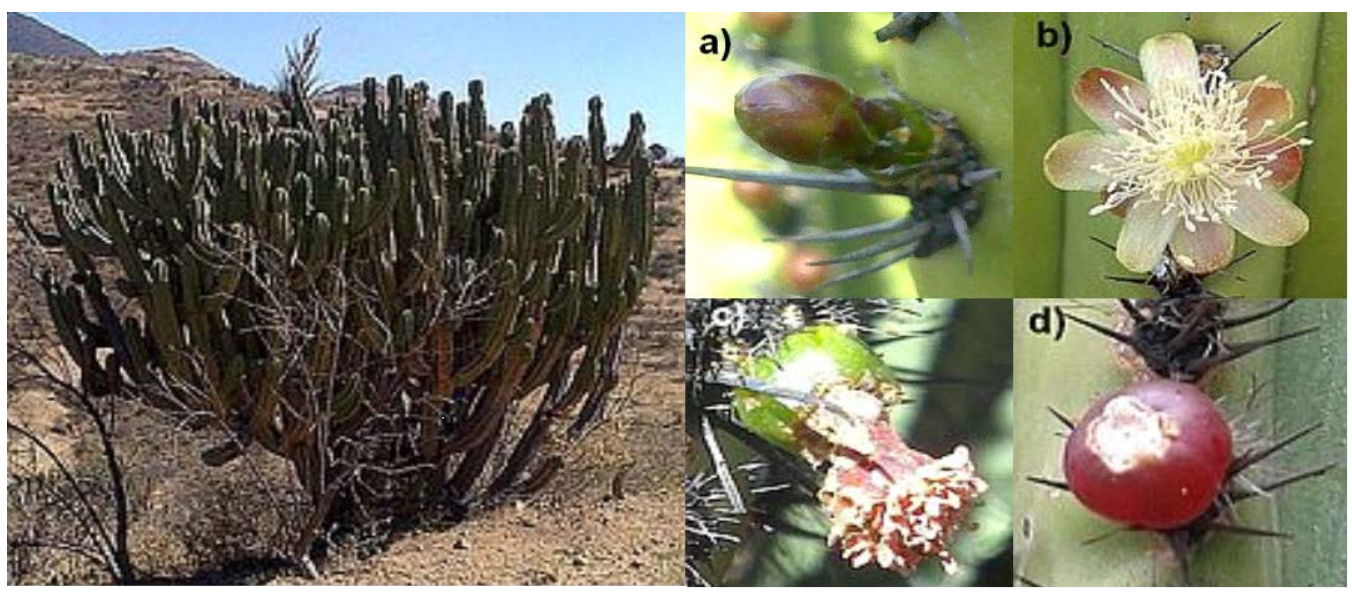

Figura 2. Myrtillocactus schenckii y sus estructuras reproductivas; a) yema, b) flor, c) fruto inmaduro, d) fruto maduro.

\section{Determinación de la distribución circular de los frutos}

Para determinar la orientación de los frutos de $M$. schenckii, se eligieron 40 individuos adultos. La copa de cada individuo se dividió en dos sectores, norte y sur, y en cada individuo se eligieron 2 ramas de cada sector, i. e. 4 ramas en total por individuo (Figura 3a). El criterio de elección fue seleccionar aquellas ramas ubicadas al extremo norte del sector norte y al extremo sur del sector sur. En cada rama se seleccionó una costilla de referencia, con orientación de $\approx$ $0^{\circ}$ para las ramas del sector norte y de orientación $\approx 180^{\circ}$ para las del sector sur utilizando una brújula Brunton. Tomando como referencia esa costilla (costilla C1, Figura 3b), se contaron el número total de costillas en las ramas y el número total de frutos por costilla. El conteo se realizó siguiendo la dirección de las manecillas del reloj (Figura 3b). Posteriormente, a partir del acimut de referencia, se estimó la orientación de cada una de las costillas dividiendo $360^{\circ}$ entre el número total de costillas de la rama (entre 6 y 8). Alrededor de 28 costillas por individuo, 80 ramas para cada sector, cada uno de estos con aproximadamente 560 costillas en total. Estos números varían porque las ramas de $M$. schenckii normalmente presentaron 7 
costillas, pero en ocasiones se presentaron 6 u 8 por rama.

Los datos obtenidos se analizaron con estadística circular (Zar, 1999), utilizando el programa ORIANA v.4.02 (Kovach, 2013), para determinar si existe una orientación preferencial para cada una de las estructuras reproductivas por separado y en conjunto. La medición de la orientación preferencial se realizó mensualmente a los 40 individuos, de enero a junio del 2014.

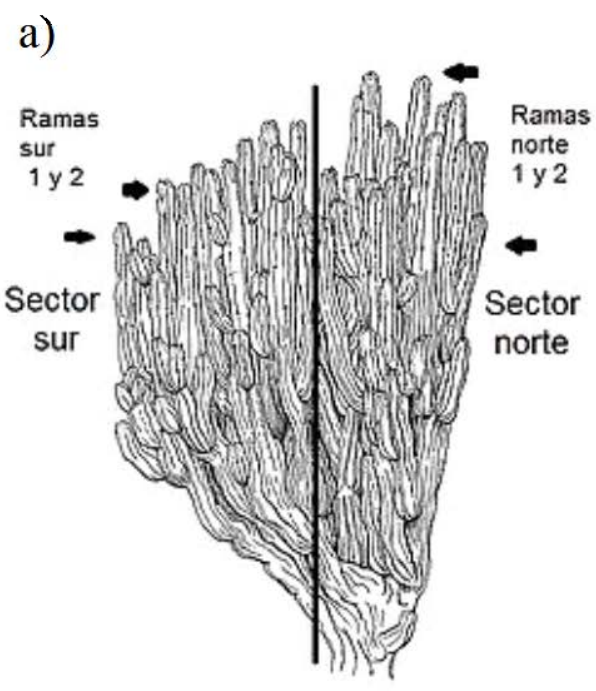

b)
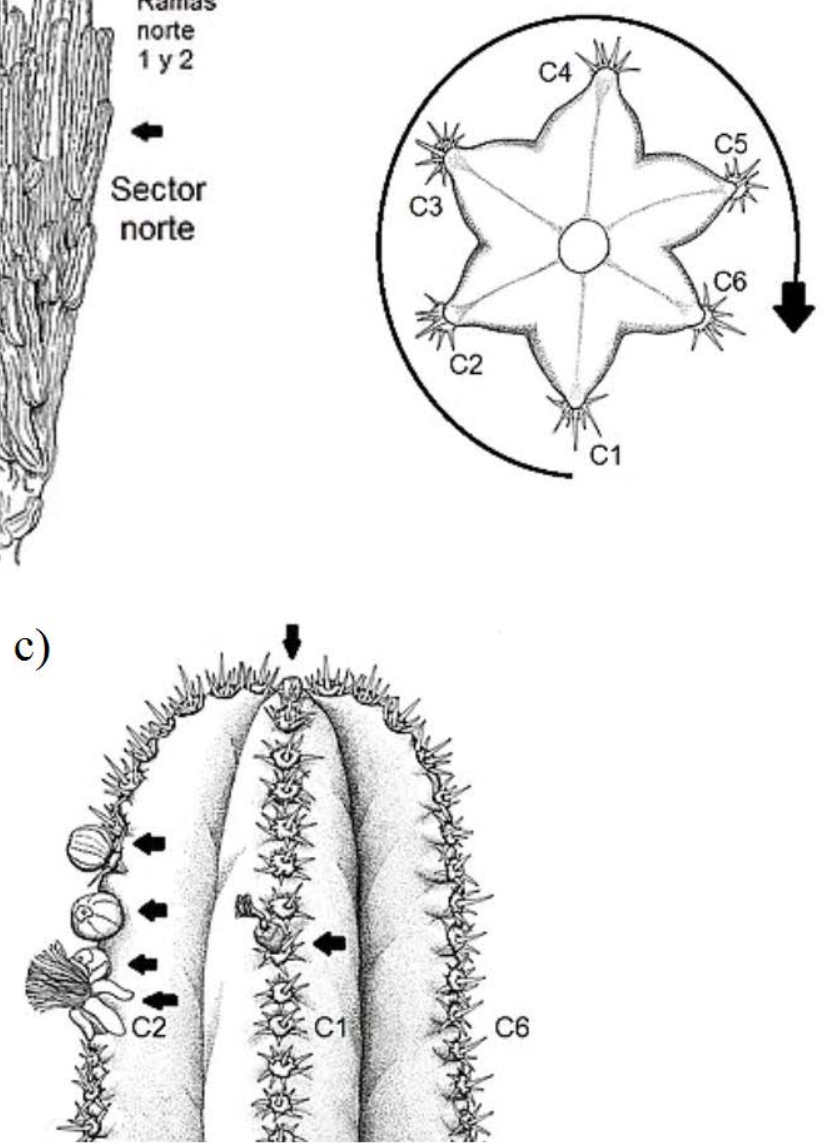

Figura 3. Conteo de estructuras de Myrtillocactus schenckii: a) división y selección de ramas por sector; b) orden del conteo de costillas; c) conteo de estructuras por costilla (en este caso la costilla C1 presenta 2 frutos, mientras que la costilla C2 presenta 3 frutos y una flor, y la costilla C6 no presenta estructuras). Modificado de Bravo-Hollis (1978). 


\section{Experimentos de remoción de frutos por consumidores diurnos y nocturnos}

En abril del 2014, se seleccionaron 20 individuos adultos de $M$. schenckii, y en cada uno de ellos se eligió una rama con frutos, mismos que fueron marcados con una pequeña gota de pintura, y adyacente a este, con una pequeña marca en la costilla. Por otro lado, se colocaron trampas para frutos debajo de cada rama elegida, a fin de contar los frutos marcados que cayeran en lugar de ser consumidos (Figura 4). Durante 3 días, las ramas con los frutos marcados de 10 individuos se cubrieron durante la noche (1800 a 0600 hrs) con bolsas de tela de tul de 1m de largo con apertura de 25cm de diámetro; a las 0600 hrs se quitaban las bolsas para que las ramas estuvieran descubiertas durante el día (de 0600 a 1800 hrs). Al otro grupo de 10 individuos se les sujetó al mismo tratamiento inverso, i.e. fueron cubiertos durante el día (0600 a 1800 hrs) y expuestos para los consumidores nocturnos. Cada día del experimento, antes de cubrir la ramas, se contaron los frutos marcados, a fin de determinar cuántos habían sido removidos en el período en el que las ramas estuvieron descubiertas; asimismo se revisaron las trampas para registrar los frutos marcados que hubieran caído dentro de ellas (Figura 4). Para disminuir la probabilidad de que las estructuras reproductivas fueran consumidas por hormigas, se rodearon las bases de las ramas con tiza repelente para insectos, y se les colocaron además, bandas de cinta con doble cara adhesiva. En total se marcaron 333 frutos para el tratamiento de consumidores diurnos y 490 para el tratamiento de consumidores nocturnos.

En mayo del 2014, se repitieron los experimentos en los mismos 20 individuos, 10 diurnos y 10 nocturnos, marcándose 251 y 287 frutos, respectivamente; en la medida de lo 
posible, se seleccionaron los mismos individuos, pero siempre conservando su grupo experimental designado (diurno o nocturno). A finales de marzo y principios de abril del 2015, se repitieron los experimentos en 10 individuos ( 5 diurnos, 175 frutos marcados y 5 nocturnos, 156 frutos), respetando el grupo de aquellos individuos que se hubieran seleccionado durante los experimentos del 2014. La proporción de frutos removidos respecto al total de frutos marcados $(p)$ entre tratamientos (diurnos y nocturnos) fueron comparadas con pruebas de $Z$ para comparar dos proporciones (Zar, 1999).

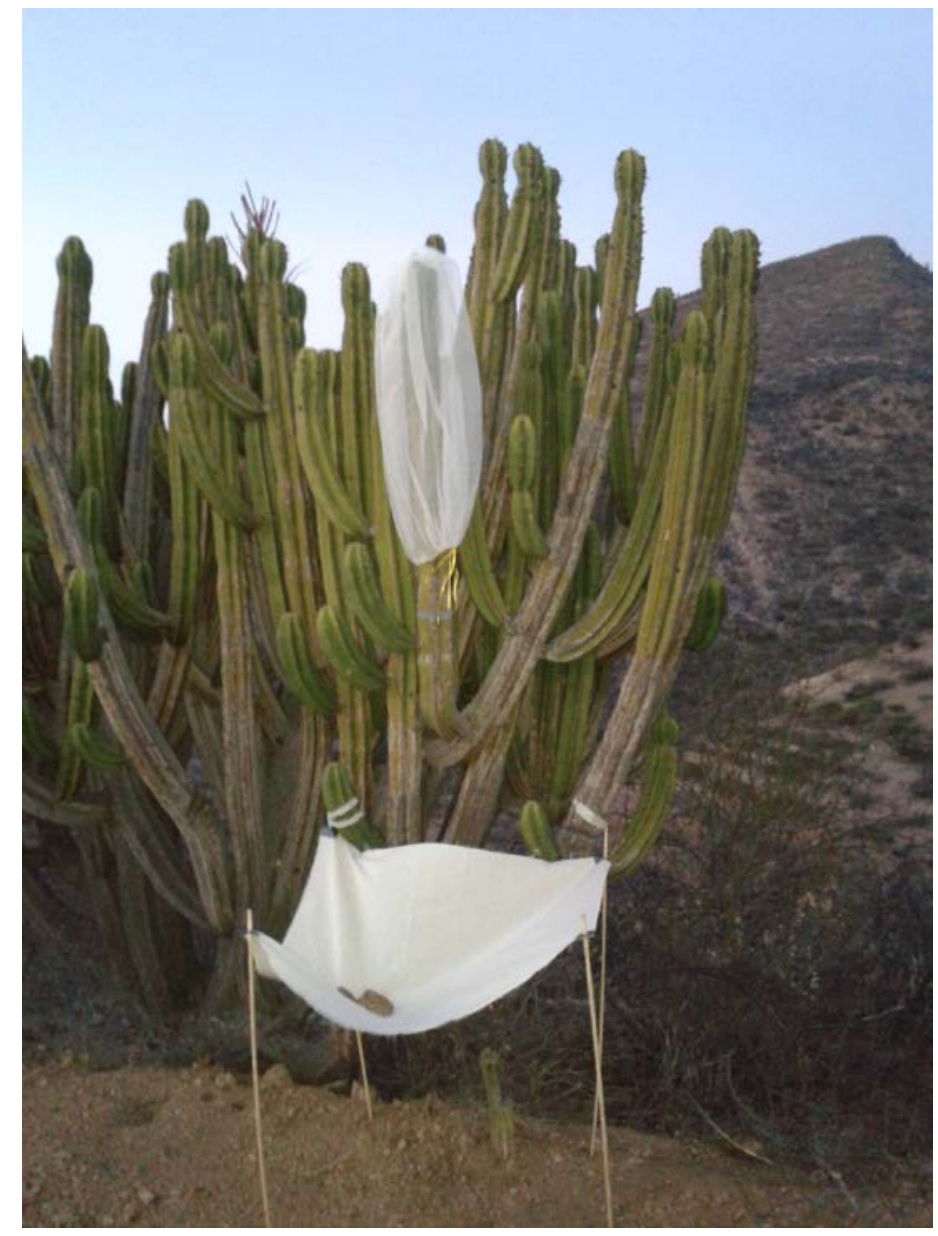

Figura 4. Rama de un individuo de Myrtillocactus schenckii cubierta durante el día, acompañada por una trampa para frutos que cayeran. 
Debido a la marcada asimetría de la distribución circular de los frutos de M. schenckii detectada durante el trabajo de campo (i. e., muy baja frecuencia de frutos en las ramas del sector norte y en las costillas norte de las ramas), no fue posible llevar a cabo las cuantificaciones para evaluar las preferencias de los frugívoros por los frutos producidos en costillas con orientaciones norte y sur. No obstante se realizaron observaciones para identificar a las aves consumidoras de los frutos de esta especie.

\section{Identificación de las aves que consumen frutos de Myrtillocactus schenckii}

En abril y mayo 2015, se eligió un punto de observación para la identificación y conteo de las aves que se alimentan de los frutos y semillas de $M$. schenckii, durante los picos de actividad de las aves (0700 a 1100 hrs y de 1500 a 1800 hrs). Estas observaciones se realizaron durante 8 días (56 horas de observación) con la ayuda de binoculares Kalimar de resolución 10 x 50, mientras que la identificación se realizó con una guía de campo (Peterson y Chalif, 2008). En cada caso, se anotó si un individuó, en su visita, consumió frutos o semillas de M. schenckii.

\section{RESULTADOS}

\section{Determinación de la distribución circular de los frutos}

Los resultados revelaron que las estructuras reproductivas de $M$. schenckii muestran una marcada orientación preferencial hacia el sur (Tablas 1 y 2). La media angular osciló de 185², en enero, a $222^{\circ}$ en junio (Tablas 1 y 2). En todos los casos, la distribución circular de las estructuras reproductivas difiere significativamente de la uniformidad (Tabla 2). Solo se 
registraron yemas florales y flores en enero y febrero (Tablas 1 y Figura 5). El mayor registro de yemas florales y flores ocurrió en el mes de enero, mientras que el de los frutos en febrero (Tablas 1 y 2, Figuras 5 y 6). De igual forma, es evidente que la mayor cantidad de frutos se registró en el sector sur de los individuos (Tabla 3, Figuras 5 y 6). En los cinco meses considerados en el presente estudio, el número de frutos contabilizados en el sector sur de los individuos siempre fue mayor que los registrados en el sector norte (Tabla 3). En promedio, en el sector sur se producen significativamente más frutos que en el sector norte $\left(t_{(2)}\right.$-pareada $=$ 2.13, g.l. $=4, P=0.0210$ ). Estas diferencias fueron, en promedio, del orden de 2.2359 veces más frutos en el sector sur con respecto al sector norte (Tabla 3).

Tabla 1. Estadísticos circulares básicos y prueba de uniformidad de las orientaciones de las yemas florales y flores de Myrtillocactus schenckii en los meses de Enero y Febrero de 2014. Linf I.C 95\% = límite inferior del intervalo de confianza del 95\% y Lsup I.C 95\% = límite inferior del intervalo de confianza del 95\%.

\begin{tabular}{lcccc}
\hline & \multicolumn{2}{c}{ Enero } & \multicolumn{2}{c}{ Febrero } \\
\hline & Yemas & Flores & Yemas & Flores \\
\hline Número de frutos $(n)$ & 2764 & 305 & 564 & 80 \\
Media circular $(\mu)$ & $190.931^{\circ}$ & $195.981^{\circ}$ & $204.131^{\circ}$ & $188.977^{\circ}$ \\
Longitud de vector medio $(r)$ & 0.523 & 0.563 & 0.495 & 0.49 \\
Concentración & 1.221 & 1.367 & 1.136 & 1.122 \\
Varianza circular & 0.477 & 0.437 & 0.505 & 0.51 \\
Desviación estándar circular & $65.269^{\circ}$ & $61.404^{\circ}$ & $67.958^{\circ}$ & $68.418^{\circ}$ \\
Error estándar circular & $1.364^{\circ}$ & $3.739^{\circ}$ & $3.218^{\circ}$ & $8.639^{\circ}$ \\
Linf I.C 95\% & $188.256^{\circ}$ & $188.65^{\circ}$ & $166.722^{\circ}$ & $172.042^{\circ}$ \\
Lsup I.C 95\% & $193.606^{\circ}$ & $203.311^{\circ}$ & $211.232^{\circ}$ & $205.912^{\circ}$ \\
Prueba de uniformidad de & $<0.0001$ & $<0.0001$ & $<0.0001$ & $<0.0001$ \\
Rayleigh $(p)$ & & & & \\
\hline
\end{tabular}


Tabla 2. Estadísticos circulares básicos y prueba de uniformidad de las orientaciones de los frutos de Myrtillocactus schenckii durante 2014. Linf I.C 95\% = límite inferior del intervalo de confianza del 95\% y Lsup I.C 95\% = límite inferior del intervalo de confianza del 95\%.

\begin{tabular}{|c|c|c|c|c|c|}
\hline & Enero & Febrero & Abril & Mayo & Junio \\
\hline Número de frutos $(n)$ & 5172 & 6847 & 5833 & 2457 & 987 \\
\hline Media circular $(\mu)$ & $182.453^{\circ}$ & $186.017^{\circ}$ & $194.91^{\circ}$ & $205.262^{\circ}$ & $222.055^{\circ}$ \\
\hline Longitud de vector medio $(r)$ & 0.583 & 0.562 & 0.557 & 0.561 & 0.551 \\
\hline Concentración & 1.441 & 1.362 & 1.346 & 1.359 & 1.324 \\
\hline Varianza circular & 0.417 & 0.438 & 0.443 & 0.439 & 0.449 \\
\hline Desviación estándar circular & $59.522^{\circ}$ & $61.528^{\circ}$ & 61.962 & 61.602 & $62.554^{\circ}$ \\
\hline Error estándar circular & $0.869^{\circ}$ & $0.792^{\circ}$ & $0.866^{\circ}$ & $1.324^{\circ}$ & $2.135^{\circ}$ \\
\hline Linf I.C 95\% & $180.749^{\circ}$ & $184.466^{\circ}$ & $193.212^{\circ}$ & $202.667^{\circ}$ & $217.869^{\circ}$ \\
\hline Lsup I.C 95\% & $184.157^{\circ}$ & $187.569^{\circ}$ & $196.609^{\circ}$ & $207.856^{\circ}$ & $226.247^{\circ}$ \\
\hline $\begin{array}{l}\text { Prueba de uniformidad de } \\
\text { Rayleigh }(p)\end{array}$ & $<0.0001$ & $<0.0001$ & $<0.0001$ & $<0.0001$ & $<0.0001$ \\
\hline
\end{tabular}

Tabla 3. Número total de frutos registrados por sectores en 40 individuos de Myrtillocactus schenckii durante 2014. E.E. = error estándar.

\begin{tabular}{lcc}
\hline Mes & Sector Sur & Sector Norte \\
\hline Enero & 3397 & 1775 \\
Febrero & 4710 & 2137 \\
Abril & 4222 & 1611 \\
Mayo & 1708 & 749 \\
Junio & 678 & 309 \\
\hline Promedio & 2943 & 1316.2 \\
\hline E.E. & 762.22 & 339.65 \\
\hline
\end{tabular}




\section{Yemas florales}

a)

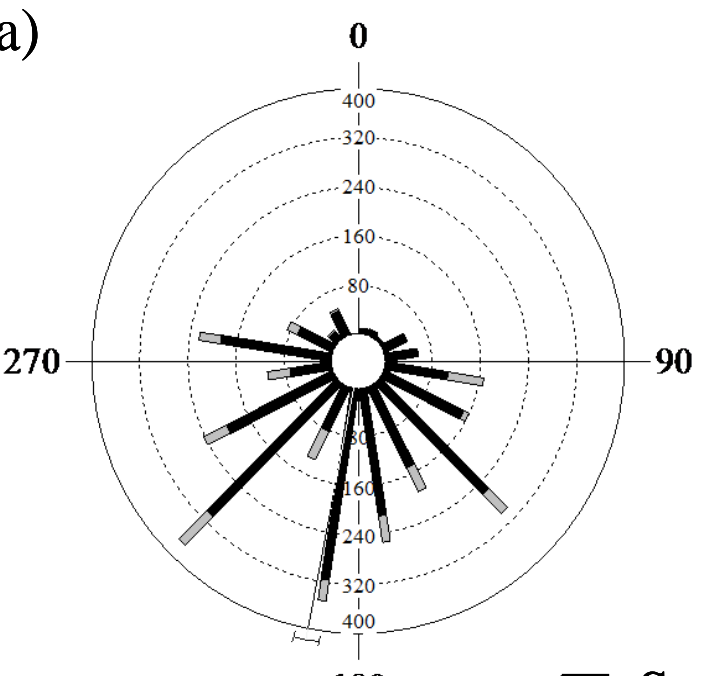

180

\section{Flores}

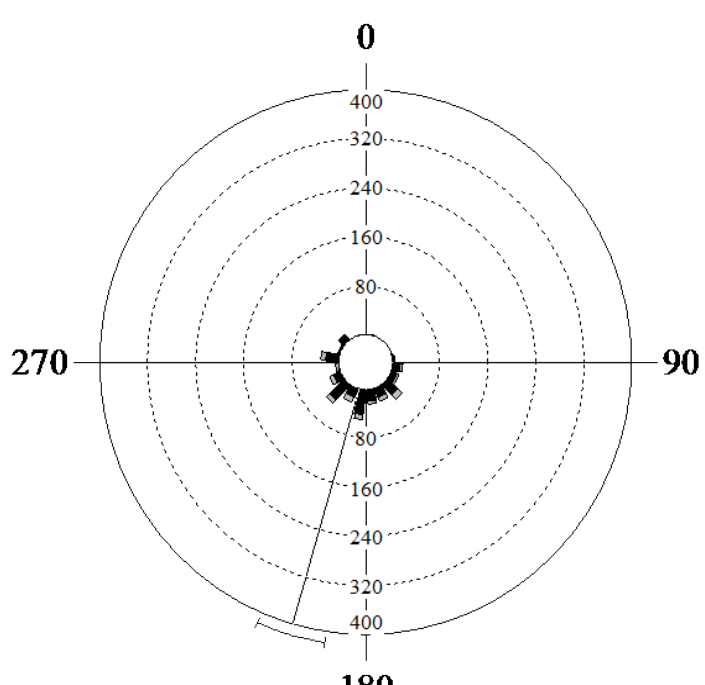

180

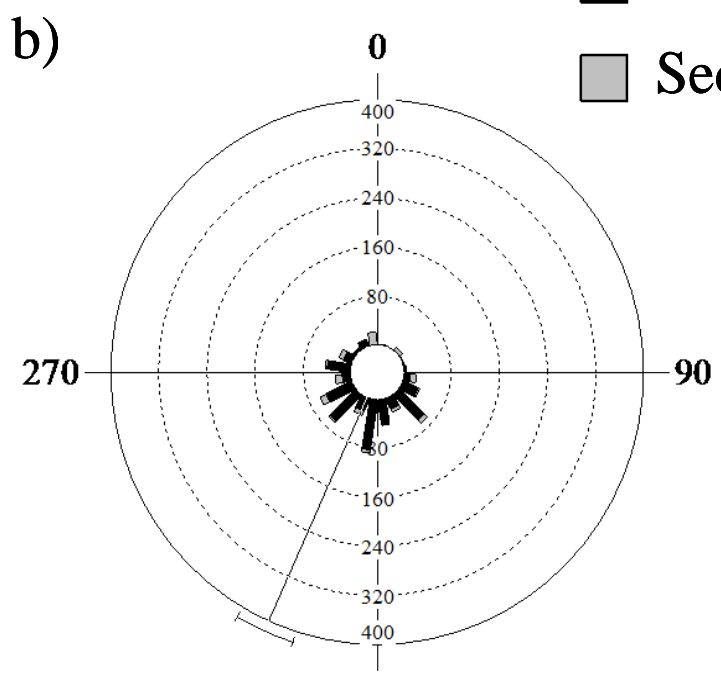

180

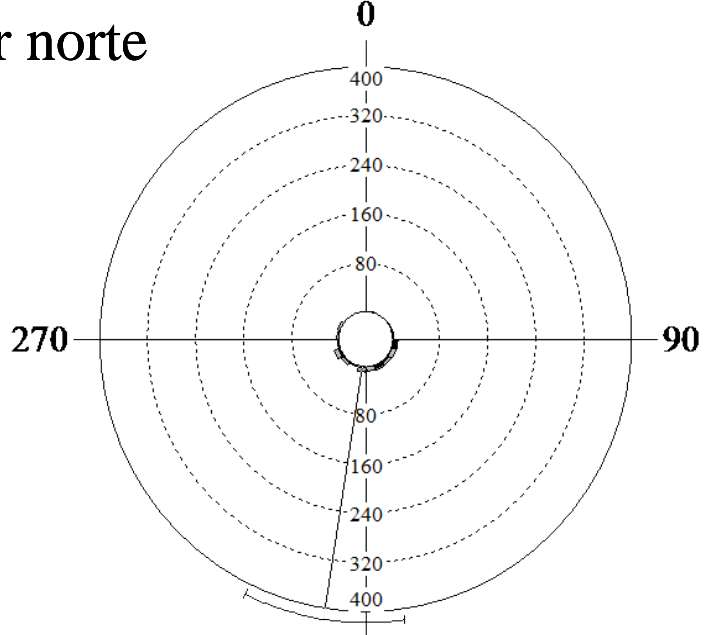

180

Figura 5. Distribución de frecuencias circulares de yemas florales y flores de Myrtillocactus schenckii, durante Enero (a) y Febrero (b) de 2014, para los sectores norte (gris) y sur (negro). En cada gráfico se muestra el intervalo de confianza del 95\% de la media circular. 


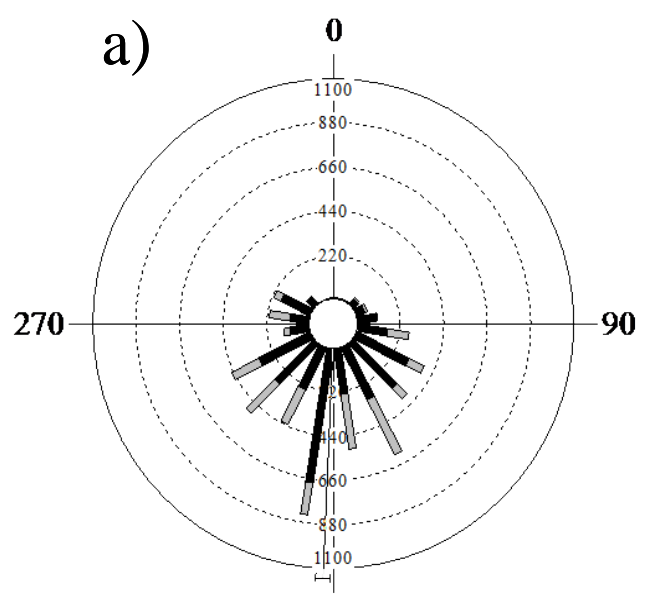

180

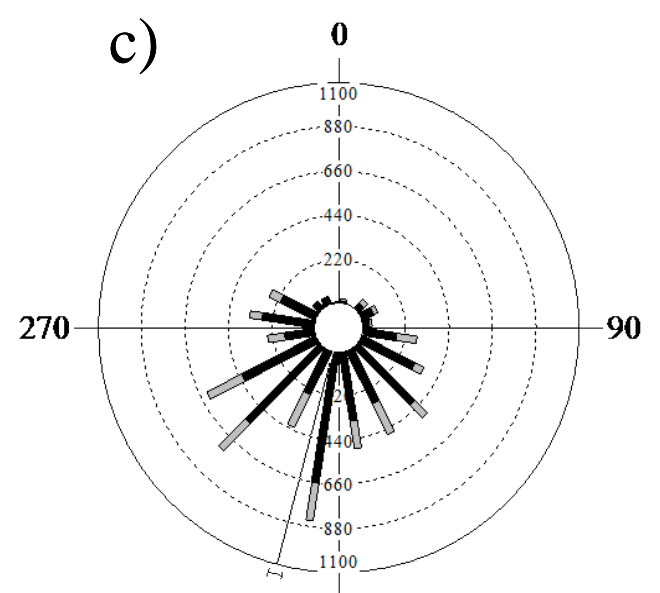

180

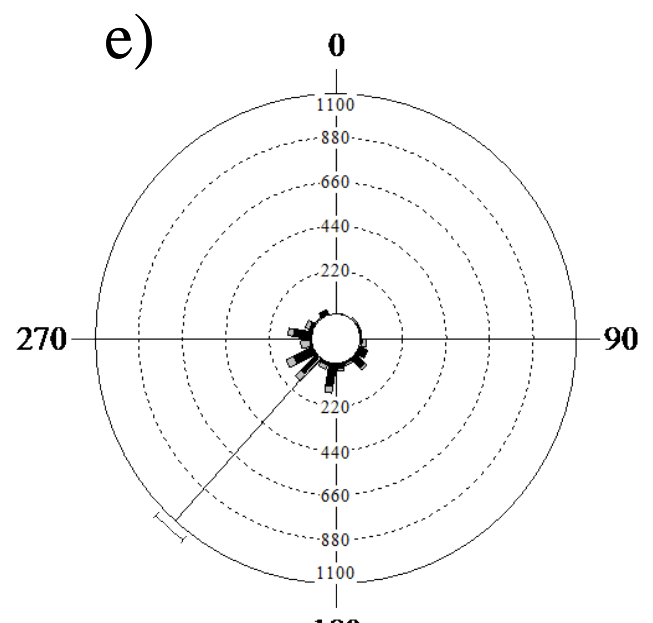

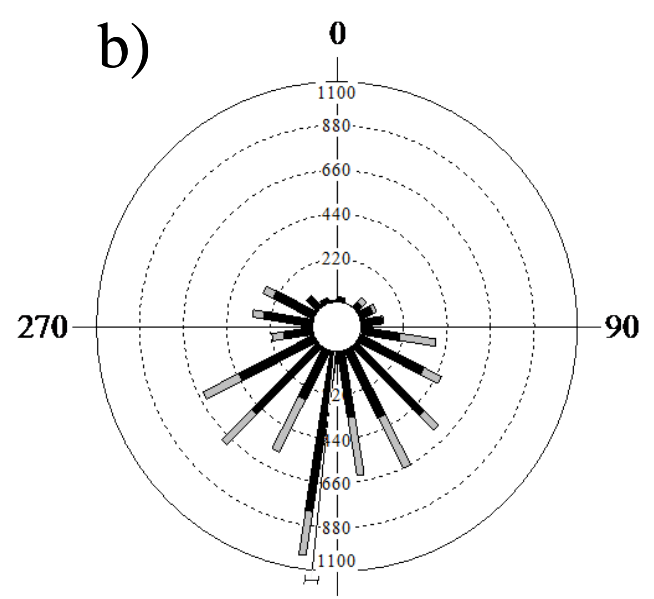

180

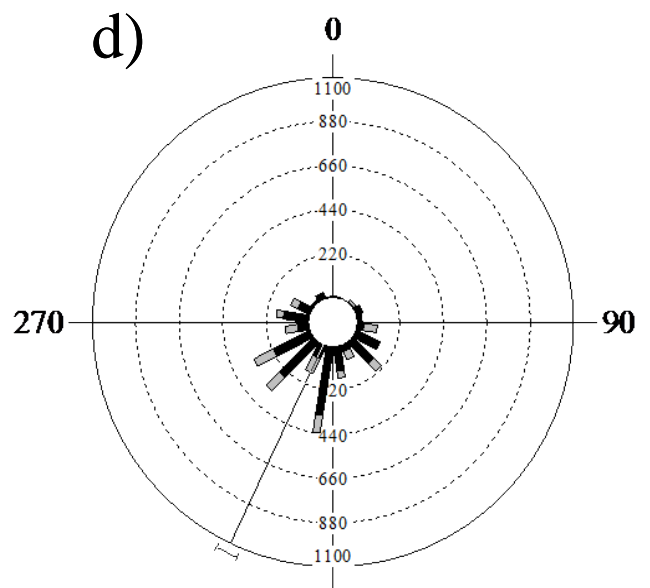

180

180

Figura 6. Distribución de frecuencias circulares de frutos de Myrtillocactus schenckii, durante Enero (a), Febrero (b), Abril (c), Mayo (d) y Junio (e) de 2014, para los sectores norte (gris) y sur (negro). En cada gráfico se muestra el intervalo de confianza del 95\% de la media circular. 


\section{Experimentos de remoción de frutos por consumidores diurnos y nocturnos}

Los experimentos de remoción de frutos revelaron que la proporción de frutos removidos fue baja $(<0.14)$ (Tabla 4). Las pruebas de Z, para comparar a los removedores diurnos contra los nocturnos, revelaron que no hay diferencias significativas entre la remoción durante el día y la noche en los experimentos de abril y mayo de 2014 ( $p=0.0681$ y 0.0749, respectivamente). Sin embargo, sí se detectaron diferencias significativas en el experimento de remoción de frutos realizado en marzo-abril del 2015 ( $p=0.0049$ ), siendo los removedores nocturnos los que más consumieron frutos en ese año (Figura 7).

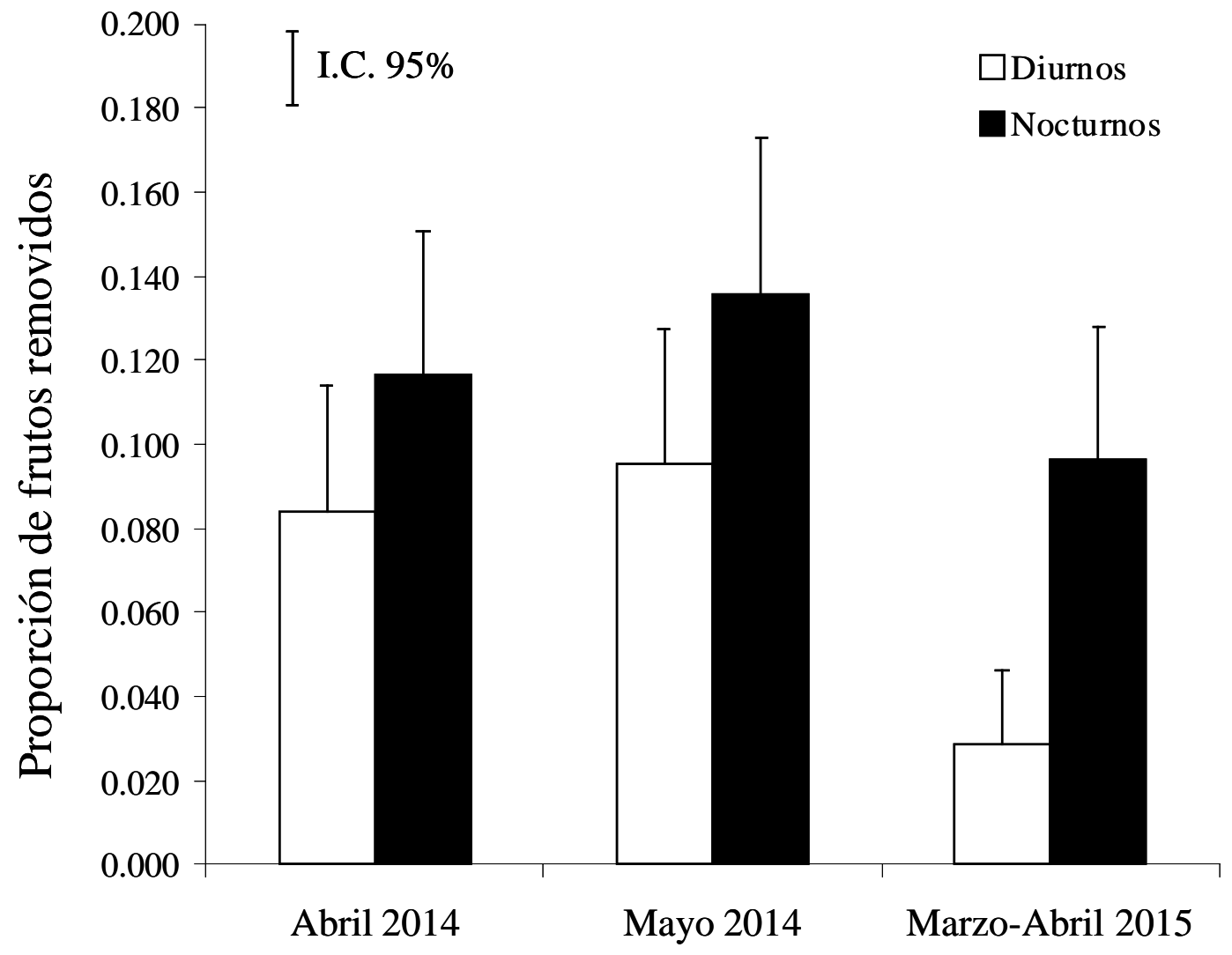

Figura 7. Proporción de frutos removidos por consumidores diurnos y nocturnos de Myrtillocactus schenckii durante los experimentos realizados en 2014 y 2015. 
Tabla 4. Resultados de los experimentos de remoción de frutos por consumidores diurnos y nocturnos de Myrtillocactus schenckii en 2014 y 2015. FM = número de frutos marcados, FR = número de frutos removidos y pfr = proporción de frutos removidos.

\begin{tabular}{|c|c|c|c|c|c|c|}
\hline \multirow{3}{*}{ Experimento } & \multicolumn{6}{|c|}{ Tratamiento } \\
\hline & \multicolumn{3}{|c|}{ Diurnos } & \multicolumn{3}{|c|}{ Nocturnos } \\
\hline & Individuo & FM & FR & Individuo & FM & FR \\
\hline \multirow{12}{*}{ Abril 2014} & 2 & 28 & 0 & 1 & 48 & 2 \\
\hline & 3 & 34 & 3 & 7 & 106 & 9 \\
\hline & 4 & 12 & 4 & 8 & 33 & 11 \\
\hline & 6 & 28 & 3 & 9 & 11 & 0 \\
\hline & 10 & 31 & 7 & 12 & 21 & 1 \\
\hline & 15 & 20 & 0 & 16 & 30 & 2 \\
\hline & 51 & 25 & 0 & 34 & 45 & 2 \\
\hline & 52 & 30 & 2 & 55 & 32 & 4 \\
\hline & 53 & 93 & 9 & 56 & 55 & 4 \\
\hline & 54 & 32 & 0 & 57 & 109 & 22 \\
\hline & Total & 333 & 28 & & 490 & 57 \\
\hline & $p f r$ & & 0.0841 & & & 0.1163 \\
\hline \multirow[t]{12}{*}{ Мауо 2014} & 2 & 36 & 0 & 1 & 31 & 1 \\
\hline & 3 & 9 & 0 & 7 & 41 & 3 \\
\hline & 4 & 27 & 4 & 8 & 25 & 3 \\
\hline & 6 & 19 & 3 & 9 & 24 & 0 \\
\hline & 10 & 33 & 0 & 12 & 9 & 2 \\
\hline & 15 & 29 & 0 & 16 & 29 & 4 \\
\hline & 51 & 23 & 11 & 38 & 25 & 8 \\
\hline & 52 & 19 & 0 & 55 & 15 & 9 \\
\hline & 53 & 27 & 4 & 56 & 45 & 3 \\
\hline & 54 & 29 & 2 & 57 & 43 & 6 \\
\hline & Total & 251 & 24 & & 287 & 39 \\
\hline & $p f r$ & & 0.0956 & & & 0.1359 \\
\hline \multirow[t]{7}{*}{ Marzo-Abril 2015} & 2 & 49 & 0 & 1 & 49 & 3 \\
\hline & 5 & 31 & 2 & 7 & 29 & 5 \\
\hline & 21 & 10 & 0 & 23 & 46 & 3 \\
\hline & 31 & 44 & 0 & 32 & 7 & 1 \\
\hline & 52 & 41 & 3 & 56 & 25 & 0 \\
\hline & Total & 175 & 5 & & 156 & 15 \\
\hline & $p f r$ & & 0.0285 & & & 0.0961 \\
\hline
\end{tabular}




\section{Identificación de las aves que consumen frutos de Myrtillocactus schenckii}

Un total de 32 individuos pertenecientes a 6 especies de aves consumieron los frutos y semillas de $M$. schenckii, entre las que destacaron Haemorhous mexicanus (35\%), Mimus polyglottos (31\%) y Melanerpes hypopolius (22\%) (Tabla 5).

Tabla 5. Especies de aves que consumen los frutos de Myrtillocactus schenckii.

\begin{tabular}{lllcc}
\hline Orden & Familia & Especie & No. de individuos & Porcentaje \\
\hline Columbiformes & Columbidae & Zenaida asiatica & 1 & 3.13 \\
Piciformes & Picidae & Melanerpes hypopolius & 7 & 21.88 \\
& Mimidae & Mimus polyglottos & 10 & 31.25 \\
Passeriformes & Ptilogonidae & Phainopepla nitens & 2 & 6.25 \\
& Cardinalidae & Passerina versicolor & 1 & 3.13 \\
& Fringilidae & Haemorhous mexicanus & 11 & 34.38 \\
\hline
\end{tabular}

\section{DISCUSIÓN}

Los resultados del presente estudio mostraron que la distribución circular de los frutos de $M$. schenckii, en la localidad de Los Reyes Metzontla, fue significativamente distinta de la uniformidad y que la producción de frutos se concentró en las costillas con orientación sur. De igual forma, las yemas florales y flores se desarrollan preferentemente en las costillas sur. El análisis por sectores también mostró que la gran mayoría de los frutos se producen en el sector sur de los individuos de M. schenckii. Como se mencionó, esta evidente asimetría en la producción de frutos no permitió evaluar las preferencias diferenciales de los frugívoros por aquellos frutos producidos en costillas con orientaciones contrastantes (norte contra sur), correspondiente al segundo objetivo particular de esta tesis. No obstante este inconveniente, 
los experimentos para evaluar la importancia de los consumidores diurnos y nocturnos, mostraron que los consumidores nocturnos removieron tantos frutos como los diurnos en el 2014, incluso superior durante el experimento realizado en el 2015. Este resultado, contrario a lo esperado, hace necesario profundizar sobre el efecto de los consumidores nocturnos en una especie de cactus columnar que presenta el síndrome de ornitocoria.

Las estructuras reproductivas (i. e., yemas florales, flores y frutos) de M. schenckii mostraron una marcada orientación preferencial hacia el sur $\left(180^{\circ} \pm 45^{\circ}\right)$. Este patrón es semejante a aquellos encontrados en otras cactáceas pertenecientes a la misma tribu (Pachycereae), tanto de zonas extratropicales como intratropicales del hemisferio norte. Por ejemplo, en Pachycereus pringlei (Tinoco-Ojanguren y Molina-Freaner, 2000) y P. pectenarboriginum (Aguilar-Gastelum y Molina-Freaner, 2015), especies extratropicales del hemisferio norte, la producción de flores y frutos ocurre principalmente en los lados orientados hacia el sur de las ramificaciones. De igual manera, pero ahora en el caso de especies intratropicales, en P. weberi (Córdova-Acosta, 2011) y M. geometrizans (RosasGarcía, 2010), flores y frutos son producidos preferentemente en las costillas con acimut sursureste.

A diferencia de lo encontrado en M. geometrizans por Rosas-García (2010), la asimetría mostrada por las estructuras reproductivas de $M$. schenckii es todavía más marcada (Tablas 1 y 2). Así por ejemplo, en las poblaciones de M. geometrizans estudiadas por RosasGarcía (2010), la longitud del vector medio ( $r$ y la concentración $(\kappa)$ de las yemas florales fueron menores a 0.390 y 0.847 , respectivamente, mientras en el caso de $M$. schenckii, los valores fueron mayores a 0.495 y 1.221, respectivamente (Tabla 1 ). 
Algunos estudios en especies de cactáceas columnares han analizado la relación de la orientación preferencial de estructuras reproductivas con la calidad de estas y con el éxito reproductivo. En el caso de $P$. weberi, la orientación preferencial está relacionada con la calidad de yemas, flores y frutos (Córdova-Acosta, 2011; Figueroa y Valverde, 2011). Por ejemplo, el número de óvulos por ovario, diámetro de las flores, la proporción de semillas/óvulos (seed set), la proporción de frutos exitosos, proporción frutos/flores (fruit set) y el peso de las semillas fue mayor en estructuras reproductivas sureñas con respecto a las norteñas (Córdova-Acosta, 2011; Figueroa y Valverde, 2011). En M. geometrizans, por otro lados, las flores con orientación hacia el sur son más grandes, producen más óvulos y presentan más anteras (Valencia-Mendoza, 2014). Aunque en este estudio no se analizó este aspecto, estudios futuros deberán explorar si la orientación preferencial de estructuras reproductivas de $M$. schenckii se relaciona con la calidad de estas.

Los experimentos de remoción mostraron diferencias entre los dos años. En el primer año (2014), no se detectaron diferencias significativas entre las remociones diurnas y nocturnas, lo que sí ocurrió en el segundo año (2015). Una posible explicación puede ser atribuida a las diferencia en las lluvias registradas en la zona de estudio en 2014 respecto a 2015. Durante 2014, las lluvias fueron de mayor intensidad que las reportadas en 2015 (CONAGUA, 2016). El déficit hídrico puede modificar ciertas características de las bayas, como el color, sabor y aroma (Deluc et al., 2009). Es posible que, las características de los frutos de M. schenckii fueran afectadas por la menor precipitación experimentada en 2015, alterando así, las preferencias de sus consumidores.

Un aspecto interesante surge al revisar el número de especies de aves que consumen frutos de M. schenckii en comparación con M. geometrizans, esta última es visitada por 25 
especies (Pérez-Villafaña y Valiente-Banuet, 2009), mientras que para M. schenckii sólo se registraron seis. Si ambas especies producen un gran número de frutos pequeños indehiscentes de color rojo intenso, con numerosas semillas, expuestos a lo largo de las costillas y ricos en carbohidratos -características que se han relacionado con el síndrome de ornitocoria- ¿por qué ocurren estas diferencias entre los frugívoros? Entre los factores que pueden influir en la baja remoción de frutos de $M$. schenckii serían los siguientes: 1) diferencias de cuatro meses entre los periodos de fructificación de $M$. geometrizans (Pérez-Villafaña y Valiente-Banuet, 2009; Blancas-Casas et al., 2009) y M. schenckii; 2) descenso en la producción de frutos en plantas silvestres en general, según lo reportan Blancas y colaboradores en el 2009, a partir de observaciones de los pobladores; 3) Diferencias en el tamaño de las semillas, que son de menor tamaño en $M$. schenckii, comparadas con las de $M$. geometrizans, lo que podría facilitar la dispersión por hormigas, fenómeno conocido como sinzoocoria, reportado por RojasAréchiga y Vázquez-Yáñez (2000) como una interacción común entre cactáceas-hormigas, consistente en la dispersión realizada por animales sin ingesta. Esto concuerda con lo observado durante los experimentos, en los que las hormigas actúan como un factor sempiterno al remover y transportar toda estructura reproductiva (yemas, flores y frutos desde inmaduros a muy maduros). Más aún, durante el experimento de 2015 la mayor remoción de frutos se llevó a cabo durante la noche, lapso en el que las aves no están presentes, por lo que estos frutos probablemente también podrían ser un recurso importante para las poblaciones de mamíferos no-voladores de hábitos nocturnos de la región. En este sentido, se han reportado especies de mamíferos como dispersores legítimos y potenciales de semillas de cactáceas columnares (Fleming y Sosa, 1994). Aunque no se observaron en el área de estudio, se ha reportado que los omnívoros Urocyon cinereoargenteus y Bassariscus astutus trepan las 
cactáceas para tomar frutos de este género (Pérez-Villafaña y Valiente-Banuet, 2009), así como especies de roedores de la zona (J. H. García-Chávez, comunicación personal). Así mismo se ha reportado que Canis latrans se alimenta de otras especies de cactáceas (Fleming y Sosa, 1994; Godínez-Álvarez et al., 2002). Finalmente, 4) aunque el manejo humano de $M$. schenckii no tiene efectos sobre su reproducción y supervivencia individual (Blancas-Casas et al., 2009), el grado de perturbación o fragmentación del hábitat modifica la heterogeneidad y complejidad de esta, así como la diversidad biótica en su conjunto, incluyendo aves y mamíferos pequeños (August, 1983). Un quinto factor podría ser la intensidad de muestreo, por lo que es necesario realizar este tipo de estudios en áreas más conservadas, y estimar si existe una diferencia significativa comparada con zonas con diferente grado de perturbación. Es recomendable llevar a cabo estudios dirigidos a observar y determinar a los mamíferos nocturnos que se alimentan de las estructuras reproductivas de $M$. schenckii, así como capturarlos para realizar análisis de sus excretas y verificar su consumo e importancia dentro de la dieta.

Las 6 especies de aves observadas están reportadas como residentes de la zona. Habitan tanto mezquitales como bosques de cactáceas columnares (Pérez-Villafaña y ValienteBanuet, 2009); dos de las tres principales especies que se alimentaron de $M$. schenckii son frugívoras-insectívoras (53\% en total), mientras que Haemorhous mexicanus, es granívoro (35\%) (Tabla 5). A diferencia de los otros consumidores, esta última especie también busca su alimento en frutos inmaduros, los cuales probablemente ya cuentan con semillas lo suficientemente maduras como para ser ingeridas.

De las tres estrategias de forrajeo propuestas por Moermond y colaboradores (1986) sólo dos se presentaron entre las aves que explotan los frutos de $M$. schenckii. El 50\% engullen 
los frutos completos (Melanerpes hypopolius, Mimus polyglottos, Phainopepla nitens), el resto picotean los frutos (Zenaida asiatica, Passerina versicolor, Haemorhous mexicanus). Estas dos técnicas de forrajeo también las presentaron las aves que remueven los frutos de $M$. geometrizans (Pérez-Villafaña y Valiente-Banuet, 2009).

\section{CONCLUSIONES}

- Las estructuras reproductivas de Myrtillocactus schenckii poseen una orientación preferencial hacia el sur.

- Es tan notable esta asimetría que no fue posible evaluar si existe una remoción preferencial de frutos producidos en las ramas o costillas sur respecto a los norteños.

- Los frutos de M. schenckii son preferentemente removidos por mamíferos nocturnos, aunque las aves también son consumidores importantes.

\section{LITERATURA CITADA}

Aguilar-Gastelum, I. y Molina-Freaner, F. 2015. Orientación de las flores de dos poblaciones norteñas de Pachycereus pecten-aboriginum (Cactaceae). Botanical Sciences 93: 1-7.

Anderson, E. F. 2001. The Cactus Family. Timber Press, Portland, Oregon, Estados Unidos.

Aguilar-Garcia, S. A. 2015. Efecto de la orientación de las flores los caracteres reproductivos de Myrtillocactus geometrizans. Tesis de Maestría en Biología. Universidad Autónoma Metropolitana-Iztapalapa. México D.F., México. 
August, V. P. 1983. The role of habitat complexity and heterogeneity in structuring tropical mammal communities. Ecology 64: 1495-1507.

Arakaki, M., Christin, P-A., Nyffeler, R., Lendel, A., Eggli, U., Ogburn, R. M., Spriggs, E., Moore, M. J. y Edwards, E. J. 2011. Contemporaneous and recent radiations of the world's major succulent plant lineages major succulent plant lineages. Proceedings of the National Academy of Sciences 108: 8379-8384.

Arias-Montes, S. Gama-López, S., Guzmán-Cruz, U. L., y Vázquez-Benítez, B. 2012. Flora del Valle Tehuacán-Cuicatlán. Fascículo 95 (Cactaceae). Universidad Nacional Autónoma de México, México, D.F., México.

Arizmendi, M. del C. y Espinosa-Monteros, A. 1996. Avifauna de los bosques de cactáceas columnares del Valle de Tehuacán, Puebla. Acta Zoológica Mexicana 67: 25-46.

Blancas-Vázquez, J. J. 2007. Manejo tradicional y variación morfológica de Myrtillocactus schenckii (J. A. Purpus) Britton y Rose en el Valle de Tehuacán, Puebla. Tesis de Posgrado en Ciencias Biologías, Universidad Nacional Autónoma de México, México, D.F., México.

Blancas, J., Casas, A., Lira, R. y Caballero, J. 2009. Traditional management and morphological patterns of Myrtillocactus schenckii (Cactaceaes) in the Tehuacan Valley, Central Mexico. Economic Botany 63: 375-387.

Bravo-Hollis, H. 1978. Las Cactáceas de México. Vol. 1. Universidad Nacional Autónoma de México, México, D.F., México.

Bravo-Hollis, H. y Scheinvar, L. 1995. El interesante mundo de las cactáceas. Fondo de Cultura Económica, México, D.F., México. 
Bustamante, E. y Búrquez, A. 2005. Frenología y biología reproductiva de las cacatáceas columnares. Cactáceas y Suculentas Mexicanas 50: 68-88.

CONAGUA. 2016. Precipitación acumulada mensual. Resúmenes mensuales de temperaturas y lluvia. http://smn.cna.gob.mx/es/climatologia/temperaturas-y-lluvias/resumenesmensuales-de-temperaturas-y-lluvias

Córdova-Acosta, E. 2011. Efecto de la orientación y caracteres de las flores en el éxito reproductivo de Pachycereus weberi en la región de Tehuacán-Cuicatlán. Tesis de Maestría en Biología. Universidad Autónoma Metropolitana-Iztapalapa, México, D.F., México.

Deluc, L. G., Quilici, D. R., Decendit, A., Grimplet, J., Wheatley, M. D., Schlauch, K. A., Mérillon, J. M., Cushman, J. C. and Cramer G.. R. 2009. Water deficit alters differentially metabolic pathways affecting important flavor and quality traits in grape berries of Cabernet Sauvignon and Chardonnay. BMC Genomics 10: 212.

Edwards, E. J. y Donoghue, M. J. 2006. Pereskia and the Origin of the Cactus Life-Form. The American Naturalist. 167: 777-793.

Edwards, E. J., Nyffeler, R. y Donaghue, M. J. 2005. Basal cactus phylogeny: implications of Pereskia (Cactaceae) paraphyly for the transition to the cactus life form. American Journal of Botany 92: 1177-1188.

Figueroa-Castro, D. M. y Valverde, P. L. 2011. Flower orientation in Pachycereus weberi (Cactaceae): Effects on ovule production, seed production and seed weight. Journal of Arid Environments 75: 1214-1217. 
Fleming, T. H. y Sosa, V. 1994. Effects of nectarivorous and frugivorous mammals on reproductive success of plants. Journal of Mammalogy 75: 845-851.

García, E. 2006. Modificaciones al sistema de clasificación climática de Köppen. 6ta edición. Instituto de Geografía. Universidad Nacional Autónoma de México, México, D.F. México.

Gibson, A. C. y Nobel, P. S. 1986. The cactus primer. Harvard University Press, Cambridge, Massachusetts, Estados Unidos.

Godínez-Álvarez, H., Valiente-Banuet, A. y Rojas-Martínez, A. 2002. The role of seed disperser in the population dynamics of the columnar cacti Neobuxbaumia tetetzo. Ecology 83: 2617-2629.

González-Medrano, F. 2012. Las zonas áridas y semiáridas de México y su vegetación. Secretaría del Medio ambiente y Recursos Naturales, Instituto Nacional de Ecología, México, D.F., México.

Herce, M. F., Martorell, C., Alfonso-Fernández, C., Váldez Villareal Boullosa L. F. y Meave, J. A. 2014. Stem tilting in the inter-tropical cactus Echinocactus platyacanthus: an adaptive solution to the trade-off between radiation acquisition and temperature control. Plant Biology 16: 571-577.

Hernández-López, D., Vaillant, F., Reynoso-Camacho, R., y Guzmán-Maldonado, S. H. 2008. Myrtillocactus (cactaceae): botanical, agronomic, physicochemical and chemical characteristics of fruits. Fruits 63: 269-276.

Howe, H. F. y Miriti, M. N. 2004. When seed dispersal matters. BioScience 54: 651-660. 
Johnson, D. S. 1924. The influence of insolation on the distribution and on developmental sequence of flowers of giant cactus of Arizona. Ecology 5: 70-82.

Kovach, W. L. (2013) ORIANA for Windows, v.4.0.2. Kovach Computing Service, Pentraeth, Gales, Reino Unido.

Levey, D. 1987. Sugar-tasting ability and fruit selection in tropical fruit-eating birds. The Auk 104: 173-179.

Moermond, T. C., Denslow, J. S., Levey, D. J. y Santana, E. C. 1986. The influence of morphology of fruit choice in neotropical birds. Frugivores and seed dispersal. Springer. Florida, Estados Unidos. Págs.137-146.

Montiel, S. y Montaña, C. 2000. Vertebrate frugivory and seed dispersal of a Chihuahuan desert cactus. Plant Ecology 146: 221-229.

Nyffeler R. y Eggli, U. 2010. A farewell to dated ideas and concepts-molecular phylogenetics and a revised suprageneric classification of the family Cactaceae. Biodiversity and Ecology 3: 109-149.

Nobel, P. S. 1981. Influences of photosynthetical active radiation on cladode orientation, stem tilting and height of cacti. Ecology 62: 982-990.

Nobel, P. S. 1998. Environmental biology of agave and cacti. Cambridge University Press, Nueva York, Estados Unidos.

Nobel, P. S. y Loik, M. E. 1999. Form and function of cacti. Robichaux RH (ed) Ecology of Sonoran Desert plants and plant communities. University of Arizona Press, Tucson, Arizona, Estados Unidos. 
Noy-Meir, I. 1973. Deserts ecosystems: environments and producers. Annual Review of Ecology and Systematics 4: 25-51.

Ortiz-Aguilar, F. 2009. Biología reproductiva de Myrtillocactus schenckii (J. Purpus) Backeb. (Cactaceae) en poblaciones silvestres y manejadas del Valle de Tehuacán-Cuicatlán, México. Tesis de Maestría en Ciencias Biológicas. Universidad Autónoma de México, Morelia, Michoacán. México.

Ortíz, F. Stoner, K. Pérez-Negrón, E. Casas, A. 2010. Pollination biology of Myrtillocactus schenckii (Cactaceae) in wild and managed populations of the Tehuacán Valley, México. Journal of Arid Environments 74: 897-904.

Osorio-Beristain, O. Valiente-Banuet, A. Dávila, P. y Medina, R. 1996. Tipos de vegetación y diversidad $\beta$ en el Valle de Zapotitlán de las Salinas. Boletín de la Sociedad botánica de México. 59: 35-58.

Pérez-Villafaña, M. G. y Valiente-Banuet, A. 2009. Effectiveness of dispersal of an ornithocorous cactus Myrtillocactus geomerizans (cactaceae) in a patchy environment. The Open Biology Journal 2: 101-113.

Peterson, R. T. y E. L. Chalif. 2008. Aves de México: Guía de Campo. Editorial Diana, México, D.F., México.

Robinson, Trevor. 1963. The Organic Constituents of Higher Plants. Burgess Publishing, Minneapolis, Estados Unidos.

Rojas-Aréchiga, M. y Vázquez-Yáñez, C. 2000. Cactus Seed germination: a review. Journal of Arid Environments. 44:85-104. 
Rosas-García, E. M. 2010. Efecto de la orientación preferencial sobre las estructuras reproductivas y vegetativas en Myrtillocactus geometrizans. Tesis de Maestría en Biología. Universidad Autónoma Metropolitana-Iztapalapa. México, D.F., México.

Rzedowski, J. 1978. Vegetación de México. Limusa, México, D.F., México.

Rzedowski, J. 1993. Diversity and origins of the Phanerogamic Flora of Mexico. En: Ramamoorthy, T.P., R. Bye, A. Lot y J. Fa (eds.). Biological diversity of Mexico. Origins and distribution. Oxford University Press, NuevaYork, Estados Unidos.

Sandoval-Palacios, E. 2010. Patrones de variación espacio-temporal del matorral xerófilo en la subcuenca de Zapotitlán, Puebla. Tesis de Maestría en Biología. Universidad Autónoma Metropolitana-Iztapalapa. México D.F., México.

Tinoco-Ojanguren, C. y Molina-Freaner, F. 2000. Flower orientation in Pachycereus pringlei. Canadian Journal of Botany 78: 1489-1494.

Valencia-Mendoza, C. M. 2014. Efecto de la orientación de las flores en el éxito de la polinización en Myrtillocactus geometrizans (Cactaceae). Tesis de Maestría en Biología. Universidad Autónoma Metropolitana-Iztapalapa, México, D.F., México.

Valiente-Banuet, L. 1991. Patrones de precipitación en el Valle de Tehuacán, Puebla, México. Tesis de Licenciatura. Facultad de Estudios Superiores Iztacala. Universidad Nacional Autónoma de México, México.

Valiente-Banuet, A., Arizmendi, M. del C., Rojas-Martínez, A., y Domínguez-Canseco, I. 1996. Ecological relationships between columnar cacti and nectar-feeding bats in Mexico. Journal of Tropical Ecology 12: 103-119. 
Valverde, P. L., Vite, F., Pérez-Hernández, M. A. y Zavala-Hurtado, J. A. 2007. Stem tilting, pseudocephalium orientation, and stem allometry in Cephalocereus columna-trajani along a short latitudinal gradient. Plant Ecology 188: 17-27.

Welty, J. C. 1982. The life of birds. 3ra ed. Saunders College Publishing. Philadelphia, Estados Unidos.

Zar, J. H. 1999. Biostatistical Analysis. Prentice Hall, Upper Saddler River, Nueva Jersey, Estados Unidos.

Zavala-Hurtado, J. A., Vite, F. y Ezcurra. E. 1998. Stem tilting and pseudocephalium orientation in Cephalocereus columna-trajani (Cactaceae): a functional interpretation. Ecology 79: 340-348. 


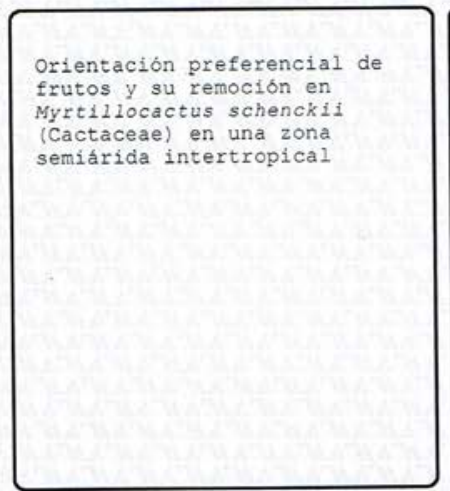

En la Ciudad de México se presentaron a las 12:00 horas del dia 2 del mes de diciembre del año 2016 en la Unidad Iztapalapa de la Universidad Autónoma Metropolitana, los suscritos miembros del jurado:

DR. MIGUEL ANGEL ARMELLA VILLALPANDO

M. EN C. FERNANDO VITE GONZALE

DR. JUAN HECTOR GARCIA CHAVEZ

DR. PABLO CORCUERA MARTINEZ DEL RIO

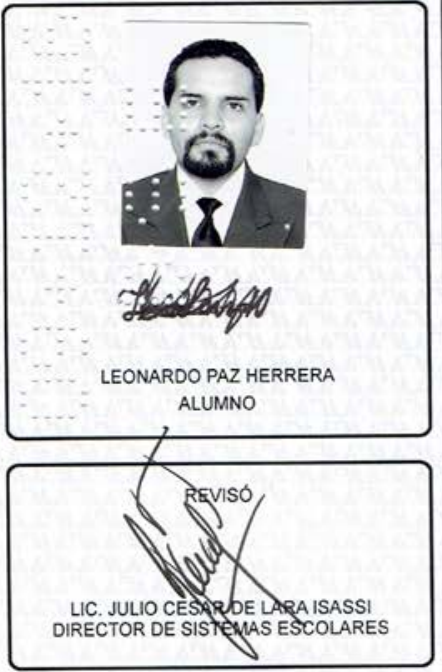

Bajo la Presidencia del primero y con carácter de Secretario el ultimo, se reunieron para proceder al Examen de Grado cuya denominación aparece al margen, para la obtención del grado de

MAESTRO EN BIOLOGIA

DE: LEONARDO PAZ HERRERA

$y$ de acuerdo con el articulo 78 fracción III del Reglamento de Estudios Superiores de la Universidad Autónoma Metropolitana, los miembros del jurado resolvieron:

\section{Aprobar}

Acto continuo, el presidente del jurado comunicó al interesado el resultado de la evaluación $y$, en caso aprobatorio, le fue tomada la protesta.

DIRECTORA DE LA DIVISIÓN DE CBS

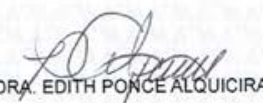

PRESIDENTE

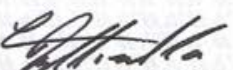

DR MIGUELANGEL ARMELLA VILLALPANDO

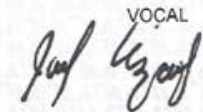

DR JUAN HECTOR GARCIA CHAVEZ 